\title{
NON-GAUSSIAN INITIAL CONDITIONS IN $\Lambda$ CDM: NEWTONIAN, RELATIVISTIC, AND PRIMORDIAL CONTRIBUTIONS
}

\author{
Marco Bruni $^{1}$, Juan Carlos Hidalgo ${ }^{1,2}$, Nikolai Meures ${ }^{1}$, and David Wands ${ }^{1}$ \\ ${ }^{1}$ Institute of Cosmology and Gravitation, University of Portsmouth, Dennis Sciama Building, Portsmouth PO1 3FX, UK \\ ${ }^{2}$ Instituto de Ciencias Físicas, Universidad Nacional Autónoma de México, Cuernavaca, Morelos 62210, Mexico \\ Received 2013 July 25; accepted 2014 February 4; published 2014 March 19
}

\begin{abstract}
The goal of the present paper is to set initial conditions for structure formation at nonlinear order, consistent with general relativity, while also allowing for primordial non-Gaussianity. We use the nonlinear continuity and Raychaudhuri equations, which together with the nonlinear energy constraint, determine the evolution of the matter density fluctuation in general relativity. We solve this equations at first and second order in a perturbative expansion, recovering and extending previous results derived in the matter-dominated limit and in the Newtonian regime. We present a second-order solution for the comoving density contrast in a $\Lambda \mathrm{CDM}$ universe, identifying nonlinear contributions coming from the Newtonian growing mode, primordial non-Gaussianity and intrinsic nonGaussianity, due to the essential nonlinearity of the relativistic constraint equations. We discuss the application of these results to initial conditions in $\mathrm{N}$-body simulations, showing that relativistic corrections mimic a non-zero nonlinear parameter $f_{\mathrm{NL}}$.
\end{abstract}

Key words: cosmology: theory - dark matter - large-scale structure of universe

\section{INTRODUCTION}

The nonlinear evolution of primordial fluctuations can be studied to learn about the physics of the early universe. In this context, the bispectrum of the cosmic microwave background radiation $(\mathrm{CMB})$ and its relation to primordial non-Gaussianity has become an important tool to study the conditions in the inflationary universe (Bartolo et al. 2004; Wands 2010; Koyama 2010; Byrnes \& Choi 2010). More recently, galaxy surveys have been used as a means to constrain primordial non-Gaussianity in large-scale structure (LSS; Dalal et al. 2008; Matarrese \& Verde 2008; Desjacques \& Seljak 2010; Giannantonio et al. 2014).

Extracting information related to the primordial nonGaussianity from the evolved matter fluctuations is, however, not trivial. The matter fields evolve nonlinearly under the action of gravity and thus, even if the primordial power spectrum is completely Gaussian, nonlinear processes would still introduce non-Gaussian correlations (Matarrese et al. 1998; Bernardeau et al. 2002; Bartolo et al. 2005; Bartolo et al. 2010). It is therefore essential to understand which kind of non-Gaussianities are induced in the matter distribution by gravity and other physical processes in order to be able to reconstruct non-Gaussianities in the primordial field.

Given the complexity of the full nonlinear equations in general relativity (GR), Newtonian equations are typically used both in analytic treatments of the nonlinear growth of structure (Peebles 1980; Bernardeau et al. 2002) and in numerical (e.g., $\mathrm{N}$-body) simulations, even when initial conditions are set on scales far larger than the causal horizon in the matter-dominated universe. This leads to the open question of how to interpret Newtonian results from a relativistic perspective, see, e.g., Chisari \& Zaldarriaga (2011); Green \& Wald (2012), also Flender \& Schwarz (2012); and Haugg et al. (2012). In this paper, we consider the relation between Newtonian dynamics of matter perturbations in a $\Lambda \mathrm{CDM}$ universe and the full general relativistic dynamics at nonlinear level. It is well known that linear matter perturbations in GR obey the same evolution equations as in Newtonian theory, and it has previously been argued that Newtonian equations accurately reproduce GR evolution up to third order (Noh \& Hwang 2004); however, we show that there are important nonlinear constraint equations which lead to corrections to the initial conditions at nonlinear orders in the perturbative expansion (Bartolo et al. 2005; Bartolo et al. 2010).

We shall study the evolution of matter density perturbations for an irrotational flow in $\Lambda \mathrm{CDM}$, presenting the solution to the GR equations for the density perturbations up to second order in the synchronous-comoving gauge. This solution is consistent with previously known separable solutions in the particular case of a matter-dominated universe $\left(\Omega_{m}=1\right)$ (Tomita 1967; Matarrese et al. 1998; Bartolo et al. 2005; Hwang et al. 2012). For $\Lambda \mathrm{CDM}$, our solution generalizes those by Bartolo et al. (2010) and Tomita (2005): Bartolo et al. (2010) solution is restricted to planar symmetry (leading to the pancake of the Zel'dovich approximation) in its Newtonian part; Tomita (2005) does not explicitly relate the second-order solution to primordial non-Gaussianity.

The derivation of the second-order solutions in GR is necessarily rather lengthy and technical in nature, so we provide here an overview of our main results.

Firstly, in Section 2, we present the nonlinear evolution equations for inhomogeneous matter in a synchronous-comoving gauge. By choosing observers comoving with the fluid fourvelocity, we are following the spirit of the covariant fluid flow approach to cosmology (Ellis 1971; Ellis et al. 2012), which makes the relativistic evolution almost identical to Lagrangian perturbations in Newtonian theory. Indeed, our starting point is the standard system of coupled evolution equations for the nonlinear density perturbation, $\delta$, and the inhomogeneous expansion of the matter flow, $\vartheta$.

In Section 3, we perform a perturbative expansion, expressing the expansion, shear, and 3 Ricci curvature in terms of spatial metric perturbations. We derive the matter density perturbations in synchronous-comoving gauge up to second perturbative order.

1. We first present the well-known first-order solutions to the linearized evolution equation, identifying a first-integral of the coupled evolution equations using the linearized energy 
constraint. This shows how the 3 Ricci curvature scalar $R^{(1)}$, which is constant, drives the growing mode of the density contrast

$$
\delta^{(1)} \propto R^{(1)}(\mathbf{x}) D_{+}(\tau),
$$

where the linearized momentum constraint ensures that $R^{(1)}$ is a constant.

2. We then repeat the same approach at second-order, using the energy constraint and the second-order 3 Ricci scalar, $R^{(2)}$. The 3 Ricci scalar is no longer constant at second order, but its time dependence is given by the momentum constraint. We split the 3 Ricci scalar into a constant part, $R_{h}^{(2)}$, and a time-dependent part, $R_{p}^{(2)}$ such that $R_{p}^{(2) \prime}=R^{(2) \prime}$. We thus split the second-order solution into a homogeneous part, obeying the same first-integral as at first order, with a growing mode solution

$$
\delta_{h}^{(2)} \propto R_{h}^{(2)}(\mathbf{x}) D_{+}(\tau),
$$

and a particular solution, including time-dependent driving terms, quadratic in first-order variables,

$$
\delta_{p}^{(2)} \propto\left(R^{(1)}(\mathbf{x})\right)^{2} D_{2+}(\tau, \Sigma(\mathbf{x})) .
$$

This solution is not in general separable since the growing mode is a function of the shape parameter, $\Sigma$ defined in Equation (67), which is in general inhomogeneous. For planar symmetry $(\Sigma=1)$ or in the matter-dominated limit $\left(\Omega_{m}=1\right)$, the solution is separable, and we find $D_{2+} \propto\left(D_{+}\right)^{2}$. This particular growing mode then dominates over the homogeneous solution at late times.

In Section 4, we discuss the relation to Newtonian results.

1. The first-order growing mode for the density perturbation, $\propto D_{+}$, whose amplitude is proportional to the comoving curvature perturbation $\mathcal{R}_{\mathrm{c}}$ in GR, is known to coincide with the first-order Newtonian solution, whose amplitude is proportional to the initial Newtonian potential $\Phi_{\mathrm{IN}}=$ $(3 / 5) \mathcal{R}_{\mathrm{c}}$.

2. The particular second-order growing mode solution, $\propto D_{2+}$, whose amplitude is proportional to $\mathcal{R}_{\mathrm{c}}{ }^{2}$, reproduces the Newtonian growing mode at second order in Lagrangian perturbation theory. We show in the Appendix that a spatial gauge transformation to Eulerian coordinates reproduces the standard Eulerian density perturbation in the matterdominated limit. This leads to a growing non-Gaussianity, but a bispectrum that vanishes in the squeezed limit.

3. The amplitude of the second-order homogeneous solution, $\propto D_{+}$, is set by initial conditions. In Newtonian theory, the Poisson equation provides a linear relation between the density and Newtonian potential at all times and at all orders in a perturbative expansion. Therefore, the initial second-order density perturbation is set by the initial second-order potential, determined, for example, by the nonlinearity parameter $f_{\mathrm{NL}}$. The second-order homogeneous solution in Newtonian theory is therefore due solely to primordial non-Gaussianity in the potential. In GR, we have nonlinear constraint equations; therefore, the second-order homogeneous solution is non-zero in general. Even in the absence of primordial nonGaussianity, in the squeezed limit we find an effective value of $f_{\mathrm{NL}}=-5 / 3$.

4. Newtonian simulations of structure formation can reproduce the "true" GR results up to second order but care needs to be taken in setting initial conditions and in identifying Newtonian and relativistic variables. Respecting the nonlinear constraint equations of GR, including both primordial non-Gaussianity and intrinsic non-Gaussianity, we can express the initial $\left(\Omega_{m \mathrm{IN}}=1\right)$ density perturbation as

$$
\begin{aligned}
\delta_{\mathrm{IN}}= & \frac{2}{3 \mathcal{H}_{\mathrm{IN}}^{2}} \nabla^{2} \Phi_{\mathrm{IN}}-\frac{4}{3 \mathcal{H}_{\mathrm{IN}}^{2}}\left[\left(f_{\mathrm{NL}}-\frac{5}{3}\right) \Phi_{\mathrm{IN}} \nabla^{2} \Phi_{\mathrm{IN}}\right. \\
& \left.+\left(f_{\mathrm{NL}}+\frac{5}{12}\right) \partial^{j} \Phi_{\mathrm{IN}} \partial_{j} \Phi_{\mathrm{IN}}\right] .
\end{aligned}
$$

We conclude in Section 5.

\section{THE NONLINEAR ANALYSIS IN SYNCHRONOUS-COMOVING GAUGE}

We will calculate the evolution of the inhomogeneous matter density in the synchronous-comoving gauge. In this section, we will present the exact, nonlinear equations. Previous derivations have been discussed in the context of a pure dust cosmology (Matarrese \& Terranova 1996; Matarrese et al. 1998; Bartolo et al. 2005) and $\Lambda$ CDM (Bartolo et al. 2010).

From a general metric, written using conformal time $\tau$,

$$
d s^{2}=a^{2}(\tau)\left[-(1+2 \phi) d \tau^{2}+2 \omega_{i} d \tau d x^{i}+\gamma_{i j} d x^{i} d x^{j}\right],
$$

the synchronous gauge (Landau \& Lifshitz 1975) is defined by setting $\phi=\omega_{i}=0$. This implies that for every observer at a fixed spatial coordinate point of the perturbed space-time, the proper time is the same as the cosmic time in the FLRW background with a scale factor of $a(\tau)$. The synchronous line element is therefore written in the form

$$
d s^{2}=a^{2}(\tau)\left[-d \tau^{2}+\gamma_{i j}(\mathbf{x}, \tau) d x^{i} d x^{j}\right],
$$

where $\gamma_{i j}(\mathbf{x}, \tau)$ is the three-metric.

We consider irrotational dust flow and choose observers comoving with the fluid. This choice implies that the fluid four-velocity can be made orthogonal to the constant time spatial hypersurfaces with metric $\gamma_{i j}$, that is, the four velocity in this gauge is $u_{\mu}=[-a, 0,0,0]$. The choice of such comoving observers is the basis of the covariant fluid approach to perturbation theory (Ellis \& Bruni 1989; Bruni et al. 1992); here, we follow the spirit of this approach and take advantage of the simplifications implicit in the choice of a set of coordinates that are simultaneously synchronous and comoving; each fluid element has coordinates assigned by its initial position (see Matarrese \& Terranova 1996). As we shall see, this approach makes the relativistic description almost identical to that of the Lagrangian perturbation theory in the Newtonian context. Actually, given that we also have a universal time, we may as well call our gauge a Lagrangian gauge (Villa et al. 2011).

The starting point of our fluid-flow description is the deformation tensor, defined as

$$
\vartheta_{v}^{\mu} \equiv a u_{; v}^{\mu}-\mathcal{H} \delta_{v}^{\mu}
$$

where we have subtracted from the derivative of the fourvelocity the isotropic background expansion, given by the conformal Hubble scalar $\mathcal{H}=a^{\prime} / a$, where a prime denotes the conformal time derivative. This deformation tensor plays a key role in our approach as it is ubiquitous in the equations that are relevant to our study. Its trace $\vartheta=\vartheta^{\alpha}{ }_{\alpha}$ represents the inhomogeneous part of the volume expansion. The traceless 
part is the matter shear tensor $\sigma_{\beta}^{\alpha}$, which represents the deviation from isotropy.

Since we have chosen $u^{\mu}$ so that it coincides with the normal to the constant time hypersurfaces, the deformation tensor (7) is purely spatial and coincides with the extrinsic curvature of the constant time slices in the conformal space-time,

$$
\vartheta_{j}^{i}=-K_{j}^{i}
$$

where $K_{j}^{i}$ can be written as (Wald 1984)

$$
K_{j}^{i} \equiv-\frac{1}{2} \gamma^{i k} \gamma_{k j}^{\prime}
$$

The continuity equation for dust follows from the energy conservation equation $u_{\alpha} T_{; \beta}^{\alpha \beta}=0$, that is,

$$
\frac{\rho^{\prime}}{\rho}=-\frac{1}{2} \gamma^{i j} \gamma_{i j}^{\prime}-3 \mathcal{H}=-\vartheta-3 \mathcal{H},
$$

where $\rho$ is the total matter density. Formally, we can solve Equation (10) in terms of the determinant, $\gamma=\operatorname{det}\left(\gamma^{i j}\right)$, to find

$$
\rho=\frac{A(\mathbf{x})}{a^{3} \sqrt{\gamma}} .
$$

In the perturbative analysis, we define the usual density contrast, $\delta$, by

$$
\rho(\mathbf{x}, \tau)=\bar{\rho}(\tau)+\delta \rho(\mathbf{x}, \tau)=\bar{\rho}(\tau)(1+\delta(\mathbf{x}, \tau)),
$$

where $\bar{\rho}$ is the background density. Then the continuity equation for the density contrast is

$$
\delta^{\prime}+(1+\delta) \vartheta=0
$$

for which the solution is

$$
\delta(\mathbf{x}, \tau)=\frac{\delta_{0}(\mathbf{x})+1}{\sqrt{\gamma(\mathbf{x}, \tau) / \gamma_{0}(\mathbf{x})}}-1 .
$$

This is the exact density fluctuation as a function of the metric, without any approximation. In practice, however, one needs to solve Einstein's equations in order to determine $\delta$ through $\gamma$.

The evolution of $\vartheta$ is given by the Raychaudhuri equation

$$
\vartheta^{\prime}+\mathcal{H} \vartheta+\vartheta_{j}^{i} \vartheta_{i}^{j}+4 \pi G a^{2} \bar{\rho} \delta=0 .
$$

We note that the two nonlinear equations (13) and (15) are formally identical to the Newtonian ones in the Lagrangian formalism (Ellis 1971; Peebles 1980) (see the discussion in Section 4.1).

In the relativistic case, Equation (15) can be obtained directly from a simple geometrical identity between the deformation tensor $\vartheta_{j}^{i}$, and the four-dimensional curvature (Ellis 1971; Wald 1984; Ellis et al. 2012), after contraction and substitution from the 00 component of the Einstein field equations (the energy constraint)

$$
\vartheta^{2}-\vartheta_{j}^{i} \vartheta_{i}^{j}+4 \mathcal{H} \vartheta+R=16 \pi G a^{2} \bar{\rho} \delta,
$$

where $R$ is the trace of $R_{j}^{i}$, the Ricci-curvature of the threemetric $\gamma_{j}^{i}$. Here we find it more useful to start from the $i j$ component of the Einstein equations, which can be written as (Wald 1984; Misner et al. 1973; Meures 2012)

$$
\vartheta_{j}^{i^{\prime}}+2 \mathcal{H} \vartheta_{j}^{i}+\vartheta \vartheta_{j}^{i}+\frac{1}{4}\left(\vartheta_{l}^{k} \vartheta_{k}^{l}-\vartheta^{2}\right) \delta_{j}^{i}+R_{j}^{i}-\frac{1}{4} R \delta_{j}^{i}=0,
$$

i.e., an evolution equation for $\vartheta_{i}^{i}$, which we will use later. Combining the trace of Equation (17),

$$
\vartheta^{\prime}+2 \mathcal{H} \vartheta+\frac{1}{4}\left[\vartheta^{2}+3 \vartheta_{j}^{i} \vartheta_{i}^{j}+R\right]=0
$$

with the energy constraint equation (16) to eliminate $R$, one obtains the dynamical equation (15).

The evolution of the deformation tensor (17) is again similar to its Newtonian counterpart, except that instead of second derivatives of the Newtonian gravitational potential, we now have the Ricci tensor for the three-metric.

Finally, we note that the $0 \mathrm{j}$ component of the Einstein field equations yields the momentum constraint

$$
\vartheta_{j \mid i}^{i}=\vartheta_{, j}
$$

where a stroke denotes a covariant derivative in the three-space with metric $\gamma_{i j}$.

\section{THE PERTURBATIVE TREATMENT}

The above treatment has been exact in the sense that we did not make any assumption about the three-metric $\gamma_{i j}$. Now we consider the case where the line element in Equation (6) is close to the spatially flat FLRW, and hence, we decompose $\gamma_{i j}$ up to the second perturbative order

$$
\begin{aligned}
\gamma_{i j} & =\delta_{i j}+\gamma_{i j}^{(1)}+\frac{1}{2} \gamma_{i j}^{(2)}+\ldots \\
& =\left(1-2 \psi^{(1)}-\psi^{(2)}\right) \delta_{i j}+\chi_{i j}^{(1)}+\frac{1}{2} \chi_{i j}^{(2)}+\ldots
\end{aligned}
$$

where the superscript denotes the order of the perturbations and

$$
\chi_{i j}=\left(\partial_{i} \partial_{j}-\frac{1}{3} \delta_{i j} \nabla^{2}\right) \chi,
$$

where $\nabla^{2}=\delta_{i j} \partial^{i} \partial^{j}$. Similarly

$$
\delta=\delta^{(1)}+\frac{1}{2} \delta^{(2)}+\ldots
$$

and equivalently for $\vartheta$ and $R$. Note that we have only included scalar quantities and that there are only two scalar degrees of freedom at each order. Vector and tensor degrees of freedom are linearly independent at first order and can consistently be set to zero, in which case they do not affect the second-order scalar perturbations. Even if they do exist at first order, they do not affect first-order density perturbations, and at second order, their effect is subdominant (Matarrese et al. 1998; Malik \& Wands 2009).

Using the equations of Section 2, we can construct differential equations governing the growth of fluctuations at each perturbative order. 


\subsection{The Background Dynamics}

In a spatially flat $\Lambda \mathrm{CDM}$ cosmology the energy constraint gives the Friedmann equation; in terms of $\mathcal{H}=a^{\prime} / a=a H$ this is

$$
3 \mathcal{H}^{2}=8 \pi G a^{2} \bar{\rho}+a^{2} \Lambda .
$$

The Raychaudhuri equation is

$$
3 \mathcal{H}^{\prime}+4 \pi G a^{2} \bar{\rho}-a^{2} \Lambda=0,
$$

and the homogeneous part of the continuity equation (10) is simply

$$
\frac{\bar{\rho}^{\prime}}{\bar{\rho}}=-3 \mathcal{H} \text {. }
$$

These equations determine the dimensionless density parameter $\Omega_{m} \equiv 8 \pi G a^{2} \bar{\rho} / 3 \mathcal{H}^{2}$ in terms of the scale factor,

$$
\Omega_{m}(\tau)=\Omega_{m 0}\left[\Omega_{m 0}+a(\tau)^{3}\left(1-\Omega_{m 0}\right)\right]^{-1},
$$

with the current value $\Omega_{m 0}=\Omega_{m}\left(\tau_{0}\right) \simeq 0.315 \pm 0.017$ (Ade et al. 2013).

Note finally that combining Equations (23) and (24), one obtains a homogeneous equation for the Hubble expansion $H$; this can be cast as

$$
H^{\prime}+\frac{3}{2} \mathcal{H} \Omega_{m} H=0
$$

and in this form it will be useful in Section 3.3.

\subsection{Kinematical, Curvature and Metric Variables at First Order}

Expanding at first order the quantities introduced in Section 2, we can relate them to the scalar metric potentials $\psi^{(1)}$ and $\chi^{(1)}$, Equations (20) and (21) (Bruni et al. 1992; Matarrese et al. 1998). The deformation tensor (8) is given by

$$
\vartheta^{(1) i}{ }_{j}=-\psi^{(1)^{\prime}} \delta_{j}^{i}+\frac{1}{2}\left(\chi^{(1)^{i}}{ }_{j}^{\prime}\right.
$$

The trace and traceless parts of $\vartheta^{(1) i}$ are, respectively, the inhomogeneous expansion scalar and the matter shear:

$$
\begin{gathered}
\vartheta^{(1)}=-3 \psi^{(1)^{\prime}}, \\
\sigma_{j}^{(1)^{i}}=\frac{1}{2}\left(\chi_{j}^{(1)^{i}}\right)^{\prime} .
\end{gathered}
$$

Additionally, expanding the 3 Ricci scalar at first order, one gets

$$
R^{(1)}=4 \nabla^{2}\left[\psi^{(1)}+\frac{1}{6} \nabla^{2} \chi^{(1)}\right] .
$$

Note that the matter shear $\sigma_{j}^{(1) i}$ and the 3 Ricci curvature of the comoving orthogonal hypersurfaces $R_{j}^{(1) i}$ are tensors that vanish in the background (the latter only in a flat background), and as such, they are gauge-invariant quantities, represented in our synchronous-comoving gauge by the right-hand side of Equations (30) and (31) (Bruni et al. 1992; Bruni et al. 1997). In this gauge, the above relations show that $\vartheta$ and $\sigma_{j}^{i}$ coincide with the expansion and the matter shear of the normal to the time slicing. ${ }^{3}$

\footnotetext{
3 Note that the shear and the 3 Ricci curvature of an arbitrary slicing are not gauge invariant (Kodama \& Sasaki 1984; Malik \& Wands 2009).
}

An important quantity in the relativistic perturbation theory of the early universe is the comoving curvature perturbation, $\mathcal{R}_{\mathrm{c}}$, the conformally flat part of the metric perturbation on comoving hypersurfaces (Lyth 1985). In terms of our metric variables, Equations (20) and (21), this is

$$
\mathcal{R}_{\mathrm{c}}=\psi^{(1)}+\frac{1}{6} \nabla^{2} \chi^{(1)},
$$

so that, from Equation (31),

$$
R^{(1)}=4 \nabla^{2} \mathcal{R}_{\mathrm{c}}
$$

We can also use the gauge-invariant potentials $\Phi$ and $\Psi$ (Bardeen 1980; Malik \& Wands 2009), which coincide with $\phi^{(1)}$ and $\psi^{(1)}$ in the Poisson gauge. In our gauge,

$$
\begin{aligned}
& \Phi=-\frac{1}{2}\left(\chi^{(1) \prime \prime}+\mathcal{H} \chi^{(1) \prime}\right) \\
& \Psi=\psi^{(1)}+\frac{1}{6} \nabla^{2} \chi^{(1)}+\frac{1}{2} \mathcal{H} \chi^{(1) \prime} .
\end{aligned}
$$

From the vanishing of the anisotropic stresses in Einstein's equations it follows that $\Phi=\Psi$ (Bardeen 1980).

Finally, we remark that the gauge-invariant potential $\Phi$ and the first-order density perturbation in our comoving gauge are related through the Poisson equation (Bardeen 1980; Bruni et al. 1992; Wands \& Slosar 2009),

$$
\nabla^{2} \Phi=\frac{3}{2} \mathcal{H}^{2} \Omega_{m} \delta^{(1)} .
$$

Another important quantity in studies of the early universe is the gauge-invariant curvature perturbation on uniform-density hypersurfaces (Malik \& Wands 2009). This is given by

$$
\zeta^{(1)} \equiv-\left(\psi^{(1)}+\frac{1}{6} \nabla^{2} \chi^{(1)}\right)-\frac{\mathcal{H}}{\rho^{\prime}} \delta \rho^{(1)}=-\mathcal{R}_{\mathrm{c}}+\frac{1}{3} \delta^{(1)} .
$$

We note from Equation (35) that $\delta^{(1)}$ is suppressed on large scales, well outside the horizon, and therefore, at early times

$$
\zeta^{(1)} \simeq-\mathcal{R}_{\mathrm{c}}
$$

In the rest of this section, we use $\mathcal{R}_{\mathrm{c}}$ to express our initial conditions in terms of this gauge-invariant quantity. ${ }^{4}$

Since our goal is to set initial conditions for structure formation at some early initial time $\tau_{\mathrm{IN}}$ in the matter-dominated era, various quantities evaluated at this time will be indicated with the sub-index $I N$.

\subsection{First-order Solutions}

We start by writing the first-order part of the continuity equation (10) as

$$
\delta^{(1)^{\prime}}+\vartheta^{(1)}=0 .
$$

The first-order expansion of the Raychaudhuri equation (15) takes the form

$$
\vartheta^{(1)^{\prime}}+\mathcal{H} \vartheta^{(1)}+\frac{3}{2} \mathcal{H}^{2} \Omega_{m} \delta^{(1)}=0
$$

\footnotetext{
$4 \delta^{(1)}$ and $\vartheta^{(1)}$ are not themselves gauge-invariant quantities, but they represent gauge-invariant variables when evaluated in our gauge (Malik \&
} Wands 2009). 
We thus obtain two equations for $\delta^{(1)}$ and $\vartheta^{(1)}$, which are decoupled from other perturbations at first order. Therefore, the solutions of the above equations solve the problem of the first-order matter density evolution. Furthermore, since there are only two scalar degrees of freedom, all other perturbations can be expressed in terms of these solutions.

Combining Equations (38) and (39), we obtain the evolution equation for the density contrast

$$
\delta^{(1)^{\prime \prime}}+\mathcal{H} \delta^{(1)^{\prime}}-\frac{3}{2} \mathcal{H}^{2} \Omega_{m} \delta^{(1)}=0 .
$$

This is the same as the Newtonian evolution equation for the matter density fluctuation (Peebles 1980).

In the relativistic formalism, we can use the energy constraint (16) to make a direct link with early universe fluctuations in terms of the 3 Ricci scalar $R$. At first order, the energy constraint (16) yields an algebraic relation between the three variables $\vartheta^{(1)}, R^{(1)}$ and $\delta^{(1)}$ :

$$
4 \mathcal{H} \vartheta^{(1)}-6 \mathcal{H}^{2} \Omega_{m} \delta^{(1)}+R^{(1)}=0 .
$$

Using Equation (38) to eliminate $\vartheta^{(1)}$, we find

$$
4 \mathcal{H} \delta^{(1)^{\prime}}+6 \mathcal{H}^{2} \Omega_{m} \delta^{(1)}-R^{(1)}=0 .
$$

On the other hand, taking the time derivative of Equation (41), and eliminating $\delta^{(1)^{\prime}}$ and $\vartheta^{(1)^{\prime}}$ using Equations (38) and (39), gives

$$
R^{(1)^{\prime}}=0 \text {. }
$$

Hence, Equation (42) is a first integral of the evolution equation (40) where the 3 Ricci scalar $R^{(1)}$ is a constant to be determined by initial conditions. Note that the momentum constraint equation (19) gives, at first order,

$$
\partial_{j}\left(6 \psi^{(1)}+\nabla^{2} \chi^{(1)}\right)^{\prime}=0
$$

i.e., $6 \partial_{j} \mathcal{R}_{\mathrm{c}}{ }^{\prime}=0$. Therefore, since Equation (43) implies that $\mathcal{R}_{\mathrm{c}}=$ const., the momentum constraint is identically satisfied, which shows the consistency of our procedure. In addition, this constraint implies that $\psi^{(1) \prime}=-(1 / 6) \nabla^{2} \chi^{(1) \prime}$, which allows us to write

$$
\vartheta_{j}^{(1) i}=\frac{1}{2} \partial^{i} \partial_{j} \chi^{(1) \prime} .
$$

We will use this last result to simplify our second-order calculations.

It is standard practice to write the general solution of Equation (40) as a linear combination of a growing mode and a decaying mode:

$$
\delta^{(1)}(\tau, \mathbf{x})=\delta_{+}^{(1)}(\mathbf{x}) D_{+}(\tau)+\delta_{-}^{(1)}(\mathbf{x}) D_{-}(\tau) .
$$

We can relate these solutions with $R^{(1)}$ through the first integral Equation (42). The decaying mode $D_{-}$is the solution to the homogeneous part of Equation (42)

$$
D_{-}^{\prime}+\frac{3}{2} \mathcal{H} \Omega_{m} D_{-}=0,
$$

i.e., the decaying mode $D_{-}$is associated with isocurvature perturbations. Comparing with Equation (27) immediately gives the solution $D_{-}=D_{-\mathrm{IN}} H / H_{\mathrm{IN}}$. The growing mode instead corresponds to the particular solution of the first integral equation (42), with $R^{(1)} \neq 0$ and a specific initial condition $\delta_{\mathrm{IN}}$ related to $R^{(1)}$ itself. This explicitly shows how the curvature perturbation drives the formation of structure. Hereafter, we discard the decaying mode and in what follows we write

$$
\delta^{(1)}(\tau, \mathbf{x})=C_{1}(\mathbf{x}) D_{+}(\tau),
$$

where $C_{1}(\mathbf{x})=\delta_{+}^{(1)}\left(\tau_{0}, \mathbf{x}\right)$, i.e., $D_{+}\left(\tau_{0}\right)=1$, and the growth factor $D_{+}(\tau)$ corresponds to the particular solution of Equation (42) written as

$$
C_{1}(\mathbf{x})\left[\mathcal{H} D_{+}^{\prime}+\frac{3}{2} \mathcal{H}^{2} \Omega_{m} D_{+}\right]-\frac{1}{4} R^{(1)}=0 .
$$

Since $R^{(1)}$ is a constant, the square bracket is also a constant, which allow us to express $C_{1}$ in terms of $R^{(1)}$ or $\mathcal{R}_{\mathrm{c}}$ :

$$
C_{1}=\frac{2}{3} \frac{\nabla^{2} \mathcal{R}_{\mathrm{c}}}{\mathcal{H}_{\mathrm{IN}}^{2} \Omega_{m \mathrm{IN}}}\left[1+\frac{2}{3} \frac{f_{1}\left(\Omega_{m \mathrm{IN}}\right)}{\Omega_{m \mathrm{IN}}}\right]^{-1} \frac{1}{D_{+\mathrm{IN}}},
$$

where one can define (Peebles 1980)

$$
f_{1} \equiv \frac{D_{+}^{\prime}}{\mathcal{H} D_{+}}=\frac{d \log D_{+}}{d \log a}=-\frac{3}{2} \Omega_{m}+\frac{\Omega_{m} a}{\delta^{(1)}} \frac{R^{(1)}}{4 \mathcal{H}_{0}^{2} \Omega_{m 0}},
$$

and the last equality is obtained using Equation (42). Note that, given that $\Omega_{m}$ is a monotonic function of $a$, Equation (26) can be inverted, so that $f_{1}=f_{1}\left(\Omega_{m}\right)$. In a matter-dominated universe with $\Omega_{m}=1$, we have $D_{+} \propto a \propto \tau^{2}$ and hence $f_{1}(1)=1$. More generally, from Equation (49) we have

$$
\left[f_{1}\left(\Omega_{m}\right)+\frac{3}{2} \Omega_{m}\right] \mathcal{H}^{2} D_{+}=\text {const },
$$

and hence

$$
\frac{D_{+}}{D_{+\mathrm{IN}}}=\left[\frac{5}{2 f_{1}\left(\Omega_{m}\right)+3 \Omega_{m}}\right] \frac{\mathcal{H}_{\mathrm{IN}}^{2}}{\mathcal{H}^{2}} .
$$

In $\Lambda$ CDM, while the universe is still matter dominated, $\Omega_{m} \simeq 1$; assuming $f_{1}=\Omega_{m}^{q}$ and $1-\Omega_{m} \ll 1$ we find, from the time derivative of the first integral Equation (52)

$$
f_{1}\left(\Omega_{m}\right) \simeq \Omega_{m}^{6 / 11},
$$

i.e., $q=0 . \overline{54}$, in agreement with Wang \& Steinhardt (1998) and Linder \& Cahn (2007). Different approximations lead to slightly different values of $q$ (Bernardeau et al. 2002; Carroll et al. 1992; Lahav et al. 1991), but the difference is negligible at the early times considered here. Henceforth, we will set initial conditions at early times in the matter-dominated era, $\Omega_{m \mathrm{IN}}=1$, such that Equation (50) simplifies to

$$
C_{1}=\frac{2}{5} \frac{\nabla^{2} \mathcal{R}_{\mathrm{c}}}{\mathcal{H}_{\mathrm{IN}}^{2}} \frac{1}{D_{+\mathrm{IN}}}
$$

and thus, we have from Equations (48), (53), and (55) the firstorder solution

$$
\delta^{(1)}=\left[f_{1}\left(\Omega_{m}\right)+\frac{3}{2} \Omega_{m}\right]^{-1} \frac{\nabla^{2} \mathcal{R}_{\mathrm{c}}}{\mathcal{H}^{2}} .
$$

For the purposes of the subsequent analysis and since we are working with a single degree of freedom, let us relate all 
our variables with the curvature perturbation $\mathcal{R}_{\mathrm{c}}$. From the continuity Equation (38) and using Equations (48) and (51) to eliminate $D_{+}^{\prime}$, we find

$$
\vartheta^{(1)}=-C_{1}(\mathbf{x}) D_{+}^{\prime}(\tau)=-\left[\frac{f_{1}\left(\Omega_{m}\right)}{f_{1}\left(\Omega_{m}\right)+(3 / 2) \Omega_{m}}\right] \frac{\nabla^{2} \mathcal{R}_{\mathrm{c}}}{\mathcal{H}} .
$$

Integrating Equation (29) gives

$$
\begin{aligned}
\psi^{(1)} & =\frac{1}{3} C_{1}(\mathbf{x}) D_{+}(\tau)+\mathcal{R}_{\mathrm{c}}(\mathbf{x}) \\
& =\frac{1}{3}\left[f_{1}\left(\Omega_{m}\right)+\frac{3}{2} \Omega_{m}\right]^{-1} \frac{\nabla^{2} \mathcal{R}_{\mathrm{c}}}{\mathcal{H}^{2}}+\mathcal{R}_{\mathrm{c}}
\end{aligned}
$$

where the constant of integration is set from the definition of $\mathcal{R}_{\mathrm{c}}$ in Equation (32). We choose initial spatial coordinates such that on large scales/early times $\psi^{(1)} \sim \mathcal{R}_{\mathrm{c}}$, consistent with the separate universe approach, where the metric approaches a manifestly FRW metric at large scales (Lyth 1985; Wands et al. 2000).

The expansion of $\mathcal{R}_{\mathrm{c}}$ in Equations (31) and (33) makes trivial the determination of $\nabla^{2} \chi^{(1)}$ in terms of this variable,

$$
\chi^{(1)}=-2 \nabla^{-2} C_{1}(\mathbf{x}) D_{+}(\tau)=-2\left[f_{1}\left(\Omega_{m}\right)+\frac{3}{2} \Omega_{m}\right]^{-1} \frac{\mathcal{R}_{\mathrm{c}}}{\mathcal{H}^{2}},
$$

$\vartheta_{j}^{(1) i}$ then follows from Equation (45). Having assumed a purely growing mode, at early times, $\tau \rightarrow 0$, the density contrast, inhomogeneous expansion, and shear are suppressed, and the only surviving perturbation is the primordial curvature perturbation, $\psi^{(1)} \rightarrow \mathcal{R}_{\mathrm{c}}$.

In this paper, we are primarily interested in connecting initial conditions at the beginning of the matter-dominated era with primordial fluctuations. In this case, although the solution Equation (56) is general, it is of practical use only if one has a solution for $f_{1}$, such as the approximation Equation (54). On the other hand, our first integral Equation (42) provides a straightforward method to obtain an explicit solution for the growing mode. Indeed the latter, as mentioned above, can be obtained as the particular solution of Equation (42). Rewriting this equation as

$$
\frac{d \delta^{(1)}}{d a}+\frac{3}{2} \frac{\Omega_{m}}{a} \delta^{(1)}=\frac{\Omega_{m}}{4 \mathcal{H}_{0}^{2} \Omega_{m 0}} R^{(1)}
$$

and using standard methods for solving first-order inhomogeneous equations, we obtain the particular solution

$$
\delta^{(1)}(a)=D_{-} \frac{R^{(1)}}{4 \mathcal{H}_{0}^{2} \Omega_{m 0}} \int_{0}^{a} \frac{\Omega_{m}}{D_{-}} d a=\nabla^{2} \mathcal{R}_{\mathrm{c}}\left(\frac{\mathcal{H}}{a}\right) \int_{0}^{a} \frac{d a}{\mathcal{H}^{3}(a)},
$$

where we used the homogeneous solution of Equation (60), i.e., the decaying mode $D_{-} \propto \mathcal{H} / a$.

To summarize, Equations (56) and (61) give the growing mode of the linear density perturbation, directly in terms of the early universe curvature fluctuation $\mathcal{R}_{\mathrm{c}}$, with no arbitrary constants.

\subsection{Second-order Solution}

We shall now derive the second-order differential equation for $\delta^{(2)}$ following the method developed in the first-order analysis.
Our method does not require solving for the second-order metric variables, which is a simplification to other works (Bartolo et al. 2010). We start with the second-order perturbative expansion of the continuity Equation Equation (10), that is

$$
\delta^{(2)^{\prime}}+\vartheta^{(2)}=-2 \delta^{(1)} \vartheta^{(1)} .
$$

The Raychaudhuri equation, Equation (15), expanded at second order is

$$
\vartheta^{(2)^{\prime}}+\mathcal{H} \vartheta^{(2)}+\frac{3}{2} \mathcal{H}^{2} \Omega_{m} \delta^{(2)}=-2 \vartheta^{(1) i}{ }_{j} \vartheta^{(1) j}{ }_{i}
$$

As before, we combine the last two equations to derive the evolution equation for $\delta^{(2)}$, finding

$$
\begin{aligned}
\delta^{(2)^{\prime \prime}} & +\mathcal{H} \delta^{(2)^{\prime}}-\frac{3}{2} \mathcal{H}^{2} \Omega_{m} \delta^{(2)}=-2 \delta^{(1)^{\prime}} \vartheta^{(1)}-2 \delta^{(1)} \vartheta^{(1)^{\prime}} \\
& -2 \mathcal{H} \delta^{(1)} \vartheta^{(1)}+2 \vartheta^{(1) i}{ }_{j} \vartheta^{(1) j}{ }_{i}
\end{aligned}
$$

We can eliminate $\vartheta^{(1)}$ and $\vartheta^{(1)^{\prime}}$ with the aid of Equations (38) and (39). We also use Equation (45) and write

$$
\begin{aligned}
\delta^{(2)^{\prime \prime}} & +\mathcal{H} \delta^{(2)^{\prime}}-\frac{3}{2} \mathcal{H}^{2} \Omega_{m} \delta^{(2)}=2\left(\delta^{(1) \prime}\right)^{2}+3 \mathcal{H}^{2} \Omega_{m}\left(\delta^{(1)}\right)^{2} \\
& +\frac{1}{2} \partial^{i} \partial_{j} \chi^{(1)^{\prime}} \partial^{j} \partial_{i} \chi^{(1)^{\prime}} .
\end{aligned}
$$

The final form of the evolution equation is found using the first-order solutions for $\delta^{(1)}$ and $\chi^{(1)}$ presented in the previous section, that is,

$\delta^{(2)^{\prime \prime}}+\mathcal{H} \delta^{(2)^{\prime}}-\frac{3}{2} \mathcal{H}^{2} \Omega_{m} \delta^{(2)}=\left[2 f_{1}^{2}+3 \Omega_{m}+2 \Sigma f_{1}^{2}\right] C_{1}^{2} D_{+}^{2}(\tau)$,

where we introduce the shape coefficient

$$
\Sigma \equiv \frac{\vartheta_{j}^{i} \vartheta_{i}^{j}}{\vartheta^{2}}=\frac{\partial_{i} \partial_{j} \mathcal{R}_{\mathrm{c}} \partial^{i} \partial^{j} \mathcal{R}_{\mathrm{c}}}{\left(\nabla^{2} \mathcal{R}_{\mathrm{c}}\right)^{2}}
$$

Instead of directly solving the evolution equation (66), let us look at a first integral of the evolution in the energy constraint equation (16), as we did in our analysis at first order. Expanded at second order, this constraint is

$$
4 \mathcal{H} \vartheta^{(2)}-6 \mathcal{H}^{2} \Omega_{m} \delta^{(2)}+R^{(2)}=2 \vartheta_{j}^{(1) i} \vartheta_{i}^{(1) j}-2 \vartheta^{(1) 2},
$$

where the second-order Ricci scalar, $R^{(2)}$, is given in terms of metric perturbations by (Matarrese et al. 1998),

$$
\begin{aligned}
\frac{1}{2} R^{(2)}= & 2 \nabla^{2}\left[\psi^{(2)}+\frac{1}{6} \nabla^{2} \chi^{(2)}\right]+6\left(\nabla \psi^{(1)}\right)^{2} \\
& +16 \psi^{(1)} \nabla^{2} \psi^{(1)}+4 \psi^{(1)} \partial_{l} \partial_{j} \chi^{(1) l j}-2 \partial_{j} \partial_{k} \psi^{(1)} \chi^{(1) j k} \\
& +\chi^{(1) j k} \nabla^{2} \chi_{j k}^{(1)}-2 \chi^{(1) j k} \partial_{l} \partial_{k} \chi^{(1) l}{ }_{j}-\partial_{l} \chi^{(1) l k} \partial_{j} \chi^{(1) j}{ }_{k} \\
& +\frac{3}{4} \partial_{k} \chi^{(1) l j} \partial^{k} \chi_{l j}^{(1)}-\frac{1}{2} \partial_{k} \chi^{(1) l j} \partial_{l} \chi_{j}^{(1) k},
\end{aligned}
$$

an equation purely dictated by geometry.

Combining Equation (68) with the continuity equation (62) to eliminate $\vartheta^{(2)}$, we obtain

$4 \mathcal{H} \delta^{(2)^{\prime}}+6 \mathcal{H}^{2} \Omega_{m} \delta^{(2)}-R^{(2)}=2 \vartheta^{(1) 2}-2 \vartheta_{j}^{(1) i} \vartheta_{i}^{(1) j}-8 \mathcal{H} \delta^{(1)} \vartheta^{(1)}$. 
As we saw from the first-order analysis Equations (42) and (43), the equation for $\delta^{(2)}$ is coupled with an equation for $R^{(2)}$. At first order, $R^{(1)}$ is conserved and Equation (42) is a first integral of Equation (40). At second order, the time derivative of Equation (68) can be reduced to

$$
R^{(2)^{\prime}}=-4 \vartheta^{(1)^{i}}{ }_{j} R^{(1)}{ }_{i}^{j}=-2\left[\partial^{i} \partial_{j} \chi^{(1)^{\prime}} \partial^{j} \partial_{i} \mathcal{R}_{\mathrm{c}}+\nabla^{2} \chi^{(1) \prime} \nabla^{2} \mathcal{R}_{\mathrm{c}}\right]
$$

One can also derive Equation (71) by taking the time derivative of Equation (69) for $R^{(2)}$ and using the momentum constraint Equation (19).

Given the correspondence between the left-hand sides of Equations (70) and (71) to their first-order equivalents, Equations (42) and (43), we solve the coupled system of these equations by separating the solution as follows:

$$
\delta^{(2)}=\delta_{h}^{(2)}+\delta_{p}^{(2)}, \quad R^{(2)}=R_{h}^{(2)}+R_{p}^{(2)},
$$

where $R_{h}^{(2)}$ is the constant solution to the homogeneous part of Equation (71) and $R_{p}^{(2)}$ is the particular solution, and $\delta_{h}^{(2)}$ is the solution of Equation (70) that is generated by assuming $R_{h}^{(2)}$ as the only source term, see Equation (85).

\subsubsection{The Particular Solution}

The particular solution of the inhomogeneous system is obtained by integrating Equation (71) directly, yielding

$$
R_{p}^{(2)}=-2\left[\partial^{i} \partial_{j} \chi^{(1)} \partial^{j} \partial_{i} \mathcal{R}_{\mathrm{c}}+\nabla^{2} \chi^{(1)} \nabla^{2} \mathcal{R}_{\mathrm{c}}\right]
$$

This contributes to the nonlinear driving terms in Equation (70), and thus the particular part of the solution. Using Equation (59) for $\chi^{(1)}$, we can write Equation (70) as

$$
4 \mathcal{H} \delta_{p}^{(2) \prime}+6 \mathcal{H}^{2} \Omega_{m} \delta_{p}^{(2)}=\left(\nabla^{2} \mathcal{R}_{\mathrm{c}}\right)^{2} S(\tau, \Sigma),
$$

where $S(\tau, \Sigma)$ is a function of time and the shape coefficient $\Sigma$ introduced in Equation (67),

$$
S(\tau, \Sigma)=\frac{2\left[\left(2 f_{1}+3 \Omega_{m}\right)(1+\Sigma)+f_{1}^{2}(1-\Sigma)+4 f_{1}\right]}{\left(f_{1}+\frac{3}{2} \Omega_{m}\right)^{2} \mathcal{H}^{2}} .
$$

Equation (74) is an inhomogeneous linear ordinary differential equation, whose solution has a standard integral form in terms of the source term $\left(\nabla^{2} R_{c}\right)^{2} S(\tau, \Sigma)$. Given the factorized form of this source term, we can then write

$$
\delta_{p}^{(2)}=P(\mathbf{x}) D_{2+}(\tau, \Sigma)
$$

with $D_{2+}\left(\tau_{0}, \Sigma\right)=1$, and thus $P(\mathbf{x}) \equiv \delta_{p}^{(2)}\left(\mathbf{x}, \tau_{0}\right)$. In analogy to the first-order case Equation (51), we can now define

$$
f_{2} \equiv \frac{1}{2} \frac{D_{2+}^{\prime}}{\mathcal{H} D_{2+}}
$$

At early times, during matter domination $\left(\Omega_{m}=1\right)$, we have the solution $D_{2+} \propto\left(D_{1+}\right)^{2} \propto a^{2}$, hence $f_{2}(1, \Sigma)=1$. In this limit, using Equation (76) in Equation (74) gives

$$
P(\mathbf{x})=\frac{2(5+2 \Sigma)}{7}\left(\frac{D_{+\mathrm{IN}}^{2}}{D_{2+I N}}\right) C_{1}^{2}(\mathbf{x})
$$

More generally, Equation (74) can be written as

$$
\begin{aligned}
& {\left[\frac{4 f_{2}+3 \Omega_{m}}{7}\right] \frac{D_{2+}(\tau, \Sigma)}{D_{2+I N}}} \\
& \quad=\left[\frac{\left(2 f_{1}+3 \Omega_{m}\right)(1+\Sigma)+f_{1}^{2}(1-\Sigma)+4 f_{1}}{2(5+2 \Sigma)}\right] \frac{D_{+}^{2}(\tau)}{D_{+\mathrm{IN}}^{2}} .
\end{aligned}
$$

Thus, the second-order particular solution equation (76) can be written as

$$
\delta_{p}^{(2)}=\left[\frac{\left(2 f_{1}+3 \Omega_{m}\right)(1+\Sigma)+f_{1}^{2}(1-\Sigma)+4 f_{1}}{4 f_{2}+3 \Omega_{m}}\right]\left(\delta^{(1)}\right)^{2},
$$

or, using Equation (56) and substituting in for the shape coefficient equation (67),

$$
\begin{aligned}
& \delta_{p}^{(2)}= \\
& \frac{\left(6 f_{1}+f_{1}^{2}+3 \Omega_{m}\right)\left(\nabla^{2} \mathcal{R}_{\mathrm{c}}\right)^{2}+\left(2 f_{1}-f_{1}^{2}+3 \Omega_{m}\right) \partial^{i} \partial_{j} \mathcal{R}_{\mathrm{c}} \partial_{i} \partial^{j} \mathcal{R}_{\mathrm{c}}}{\left(4 f_{2}+3 \Omega_{m}\right)\left(f_{1}+\frac{3}{2} \Omega_{m}\right)^{2} \mathcal{H}^{4}} .
\end{aligned}
$$

For $\Omega_{m} \simeq 1$, we can set $f_{2}\left(\Omega_{m}, \Sigma\right) \simeq \Omega_{m}^{p}$ (as we did at first order), where $p=p(\Sigma)$ can be determined by taking the time derivative of the logarithm of Equation (79),

$$
\frac{4 p+3}{7} \Omega_{m}^{\prime}+2 \mathcal{H} \Omega_{m}^{p} \simeq \frac{(48 / 11)+3(1+\Sigma)}{10+4 \Sigma} \Omega_{m}^{\prime}+2 \mathcal{H} \Omega_{m}^{6 / 11},
$$

where we have used Equation (54) for $f_{1}$. The time dependence of $\Omega_{m}$, at first order in $1-\Omega_{m}$, is given from the background equation (26); $\Omega_{m}^{\prime} \simeq 3 \mathcal{H}\left(\Omega_{m}-1\right)$. Thus, we obtain

$$
26 p=-\frac{15}{11}+\frac{21}{5+2 \Sigma}\left[\frac{24}{11}+\frac{3}{2}(1+\Sigma)\right] \text {. }
$$

For the special case of planar symmetry, the shape coefficient equation (67) has the value $\Sigma=1$. In this case Equation (79) directly shows that $f_{2}\left(\Omega_{m}, 1\right)=f_{1}\left(\Omega_{m}\right)$ and hence $D_{2+} \propto$ $\left(D_{1+}\right)^{2}$. The particular solution equation (81) then reduces to a remarkably simple form

$$
\delta_{p}^{(2)}=2\left(\delta^{(1)}\right)^{2}
$$

The planar case $\Sigma=1$ describes an exact solution leading to the formation of a pancake during gravitational collapse. This exact solution is an important case because it plays the role of an attractor solution in the Zel'dovich approximation in the Newtonian framework (Zel'dovich 1970; Buchert 1992; Bruni et al. 2003).

\subsubsection{The Homogeneous Solution}

For the homogeneous part of the solution, we solve the same system of coupled equations, Equations (42) and (43), as at first order,

$$
\begin{gathered}
4 \mathcal{H} \delta_{h}^{(2) \prime}+6 \mathcal{H}^{2} \Omega_{m} \delta_{h}^{(2)}-R_{h}^{(2)}=0 \\
R_{h}^{(2) \prime}=0
\end{gathered}
$$


Therefore, $R_{h}^{(2)}=$ const., and the homogeneous growing mode solution is given by $R_{h}^{(2)} \neq 0$,

$$
\delta_{h}^{(2)}(\tau, \mathbf{x})=C_{2}(\mathbf{x}) D_{+}(\tau)
$$

where $D_{+}(\tau)$ is the linear growth factor in Equation (52) or Equation (61) and

$$
C_{2}(\mathbf{x})=\frac{R_{h}^{(2)}(\mathbf{x})}{10 \mathcal{H}_{\mathrm{IN}}^{2} D_{+\mathrm{IN}}},
$$

in complete analogy with Equation (55). The second-order constant of integration, $R_{h}^{(2)}$, is derived in terms of secondorder metric variables by subtracting the particular solution, Equation (73), from the expression for $R^{(2)}$ given in Equation (69), which yields

$$
\begin{aligned}
\frac{1}{2} R_{h}^{(2)}= & 2 \nabla^{2}\left[\psi^{(2)}+\frac{1}{6} \nabla^{2} \chi^{(2)}\right]+16 \mathcal{R}_{\mathrm{c}} \nabla^{2} \mathcal{R}_{\mathrm{c}}+6 \partial^{k} \mathcal{R}_{\mathrm{c}} \partial_{k} \mathcal{R}_{\mathrm{c}} \\
& -\left[2 \partial^{k} \nabla^{2} \chi^{(1)} \partial_{k} \mathcal{R}_{\mathrm{c}}+\partial^{i} \partial_{j} \chi^{(1)} \partial^{j} \partial_{i} \mathcal{R}_{\mathrm{c}}+\nabla^{2} \chi^{(1)} \nabla^{2} \mathcal{R}_{\mathrm{c}}\right] \\
& +\frac{1}{4}\left[\partial^{i} \partial^{j} \partial^{k} \chi^{(1)} \partial_{i} \partial_{j} \partial_{k} \chi^{(1)}-\partial^{k} \nabla^{2} \chi^{(1)} \partial_{k} \nabla^{2} \chi^{(1)}\right] .
\end{aligned}
$$

\subsubsection{The Complete Solution}

The general growing-mode solution for the second-order density perturbation equation (72) can thus be written in terms of Equations (76) and (87) as

$$
\begin{aligned}
\delta^{(2)}= & P(\mathbf{x}) D_{2+}(\tau, \Sigma)+C_{2}(\mathbf{x}) D_{+}(\tau) \\
= & \frac{2(5+2 \Sigma) D_{+\mathrm{IN}}^{2}}{7 D_{2+\mathrm{IN}}} C_{1}^{2}(\mathbf{x}) D_{2+}(\tau, \Sigma) \\
& +\frac{1}{10 \mathcal{H}_{\mathrm{IN}}^{2} D_{+\mathrm{IN}}} R_{h}^{(2)}(\mathbf{x}) D_{+}(\tau) .
\end{aligned}
$$

In principle, we are free to set the constant $R_{h}^{(2)}$ at any time. In practice, we wish to determine $R_{h}^{(2)}$ in terms of the primordial metric perturbations, using Equation (89). We can do this on scales which lie outside the horizon at the start of the matter-dominated era. This limits the range of scales for which our subsequent analysis is valid; only those modes larger than the horizon at the equality of radiation and matter will obey the following initial conditions (this corresponds to wave numbers $k \gtrsim(90 \mathrm{Mpc})^{-1} h$; Lyth \& Liddle 2009). On smaller scales, we could use the output of numerical secondorder Einstein-Boltzmann codes, e.g., Pettinari et al. (2013), and construct the complete expression in Equation (89) on all scales at the start of the matter-dominated era.

The primordial curvature perturbation is commonly given in terms of the nonlinear variable $\zeta$ (Malik \& Wands 2009), such that

$$
\exp (2 \zeta)=1-2\left[\psi+\frac{1}{6} \nabla^{2} \chi\right]
$$

This extends the definition in Equation (36) to nonlinear orders. $\zeta$ is constant for adiabatic density perturbations in the longwavelength limit (Malik \& Wands 2004; Lyth et al. 2005; Langlois \& Vernizzi 2005). Since at the end of inflation all scales lie far outside the horizon, this variable is commonly used to describe the primordial curvature perturbation beyond linear order. In particular, it is used to define primordial non-Gaussianity, and the nonlinearity parameter $f_{\mathrm{NL}}$ (Lyth \& Rodríguez 2005). For local-type non-Gaussianity, we have the second-order expansion

$$
\zeta_{\mathrm{IN}}=\zeta_{\mathrm{IN}}^{(1)}+\frac{3}{5} f_{\mathrm{NL}} \zeta_{\mathrm{IN}}^{(1)^{2}}
$$

where $\zeta^{(1)}{ }_{\text {IN }}(\mathbf{x})$ is a first-order Gaussian random field due to quantum vacuum fluctuations during inflation. A Gaussian distribution of primordial perturbations from inflation then corresponds to $f_{\mathrm{NL}}=0$ (Maldacena 2003).

The nonlinear definition equation (91), expanded at second order and using Equation (92), yields

$\psi_{\mathrm{IN}}^{(2)}+\frac{1}{6} \nabla^{2} \chi_{\mathrm{IN}}^{(2)}=-\left(\frac{6}{5} f_{\mathrm{NL}}+2\right) \zeta_{\mathrm{IN}}^{(1)^{2}}=-\left(\frac{6}{5} f_{\mathrm{NL}}+2\right) \mathcal{R}_{\mathrm{c}}{ }^{2}$.

Note that we only expect the primordial perturbation $\zeta$ to remain constant to leading order in a gradient expansion. Therefore, we can only use Equation (93) to set the initial conditions on large scales and early times in the matter era, $k^{2} \ll \mathcal{H}_{\mathrm{IN}}^{2}$. Thus, combining Equations (93) and (89), we set the initial conditions for super-horizon scales at the start of the matter-dominated era

$\frac{1}{2} R_{h}^{(2)} \simeq-2 \nabla^{2}\left[\left(\frac{6}{5} f_{\mathrm{NL}}+2\right) \mathcal{R}_{\mathrm{c}}{ }^{2}\right]+16 \mathcal{R}_{\mathrm{c}} \nabla^{2} \mathcal{R}_{\mathrm{c}}+6 \partial^{k} \mathcal{R}_{\mathrm{c}} \partial_{k} \mathcal{R}_{\mathrm{c}}$

Equation (59) shows explicitly that $\chi^{(1)} \propto \mathcal{R}_{\mathrm{c}} / \mathcal{H}^{2}$, therefore, the terms involving $\chi^{(1)}$ (the second and third lines) in Equation (89) are subdominant for modes larger than the initial horizon scale, $k^{2} \ll \mathcal{H}_{\mathrm{IN}}^{2}$. In this limit, we can write the homogeneous part of the second-order density perturbation solution equations (87) as

$$
\begin{aligned}
\delta_{h}^{(2)}= & -\frac{12}{5 \mathcal{H}^{2}}\left[f_{1}\left(\Omega_{m}\right)+\frac{3}{2} \Omega_{m}\right]^{-1} \\
& \times\left\{\left(f_{\mathrm{NL}}-\frac{5}{3}\right) \mathcal{R}_{\mathrm{c}} \nabla^{2} \mathcal{R}_{\mathrm{c}}+\left(f_{\mathrm{NL}}+\frac{5}{12}\right) \partial^{j} \mathcal{R}_{\mathrm{c}} \partial_{j} \mathcal{R}_{\mathrm{c}}\right\} .
\end{aligned}
$$

This homogeneous solution illustrates how primordial nonGaussianity is transferred to the matter perturbations in a manner consistent with GR. Additionally, it shows how GR itself leads to nonlinear constraint equations which contributes to the initial non-Gaussianity of the matter density field, even if $f_{\mathrm{NL}}=0$.

Finally, we can give the complete solution, Equation (90), combining the homogeneous solution, Equation (95), and the particular solution, Equation (81). At leading order in a gradient expansion, we obtain

$$
\begin{aligned}
& \delta^{(2)}(\mathbf{x}, \tau)=-\frac{12}{5 \mathcal{H}^{2}}\left[f_{1}+\frac{3}{2} \Omega_{m}\right]^{-1} \\
& \times\left\{\left(f_{\mathrm{NL}}-\frac{5}{3}\right) \mathcal{R}_{\mathrm{c}} \nabla^{2} \mathcal{R}_{\mathrm{c}}+\left(f_{\mathrm{NL}}+\frac{5}{12}\right) \partial^{j} \mathcal{R}_{\mathrm{c}} \partial_{j} \mathcal{R}_{\mathrm{c}}\right\} \\
& +\frac{\left(6 f_{1}+f_{1}^{2}+3 \Omega_{m}\right)\left(\nabla^{2} \mathcal{R}_{\mathrm{c}}\right)^{2}+\left(2 f_{1}-f_{1}^{2}+3 \Omega_{m}\right) \partial^{i} \partial_{j} \mathcal{R}_{\mathrm{c}} \partial_{i} \partial^{j} \mathcal{R}_{\mathrm{c}}}{\left(4 f_{2}+3 \Omega_{m}\right)\left(f_{1}+\frac{3}{2} \Omega_{m}\right)^{2} \mathcal{H}^{4}},
\end{aligned}
$$

with the first line representing the primordial non-Gaussianity and GR correction, which dominate at large scales, and the last 
line corresponding to the growing Newtonian solution, which dominates on small scales.

Equation (96) agrees with the second-order density perturbation in the synchronous-comoving gauge presented in Equation (7) of Bartolo et al. (2005) in the matter-dominated limit, $\Omega_{m}=1$. On the other hand, our solution is consistent with Equation (5.1) of Tomita (1967), Equation (4.39) of Matarrese et al. (1998), and Equation (40) of Hwang et al. (2012), in the matter-dominated era for the particular choice of primordial non-Gaussianity parameter $f_{\mathrm{NL}}=-5 / 3$ (the equivalence with Hwang et al. (2012) is explicit when the solution is written in Eulerian spatial coordinates, see Appendix). We can also compare the second-order solution in a $\Lambda$ CDM cosmology with that presented by Tomita (2005), where Equation (2.22) is consistent with our result for the particular case $f_{\mathrm{NL}}=-5 / 3$. We finally comment on the result of Bartolo et al. (2010). Their solution for the second-order density perturbation is written in terms of the initial potential, $\Phi_{\mathrm{IN}}=(3 / 5) \mathcal{R}_{\mathrm{c}}$, and the linear growth factor Equation (52)

$$
D_{+}(\tau)=\left(\frac{f_{1}\left(\Omega_{m 0}\right)+\frac{3}{2} \Omega_{m 0}}{f_{1}+\frac{3}{2} \Omega_{m}}\right) \frac{\mathcal{H}_{0}^{2}}{\mathcal{H}^{2}},
$$

under the approximation $D_{2+} \propto D_{+}^{2}$, which we have seen only strictly holds during matter domination $\left(\Omega_{m}=1\right)$ or for the planar case $\Sigma=1$. In this approximation, we have

$$
\begin{aligned}
& \delta^{(2)}(\mathbf{x}, \tau)=-\frac{20}{3 \mathcal{H}_{0}^{2}}\left[f_{1}\left(\Omega_{m 0}\right)+\frac{3}{2} \Omega_{m 0}\right]^{-1} \\
& \times\left\{\left(f_{\mathrm{NL}}-\frac{5}{3}\right) \Phi_{\mathrm{IN}} \nabla^{2} \Phi_{\mathrm{IN}}+\left(f_{\mathrm{NL}}+\frac{5}{12}\right) \partial^{j} \Phi_{\mathrm{IN}} \partial_{j} \Phi_{\mathrm{IN}}\right\} D_{+}(\tau) \\
& +\frac{50}{63 \mathcal{H}_{0}^{4}}\left[f_{1}\left(\Omega_{m 0}\right)+\frac{3}{2} \Omega_{m 0}\right]^{-2} \\
& \times\left[5\left(\nabla^{2} \Phi_{\mathrm{IN}}\right)^{2}+2 \partial^{i} \partial_{j} \Phi_{\mathrm{IN}} \partial_{i} \partial^{j} \Phi_{\mathrm{IN}}\right] D_{+}^{2}(\tau) .
\end{aligned}
$$

remembering that in fact $\partial^{i} \partial_{j} \mathcal{R}_{\mathrm{c}} \partial_{i} \partial^{j} \mathcal{R}_{\mathrm{c}}=\left(\nabla^{2} \mathcal{R}_{\mathrm{c}}\right)^{2}$ when $\Sigma=1$. This expression agrees with the solution presented in Equation (45) of Bartolo et al. (2010) if we adopt their nonlinearity parameter $a_{\mathrm{NL}}=1+(3 / 5) f_{\mathrm{NL}}$.

\section{RELATION TO NEWTONIAN RESULTS}

\subsection{Equivalence of Non-linear Evolution Equations}

In the Newtonian treatment, the continuity equation in Eulerian coordinates is

$$
\frac{\partial \delta_{N}}{\partial \tau}+\nabla \cdot\left[\left(1+\delta_{N}\right) \mathbf{v}\right]=0
$$

where $\tau$ denotes conformal time, $\mathbf{v}=\mathbf{x}^{\prime}$ and $\mathbf{x}$ is the background comoving coordinate. The Euler equation, which dictates the flow evolution, is

$$
\frac{\partial \mathbf{v}}{\partial \tau}+\mathcal{H} \mathbf{v}+(\mathbf{v} \cdot \nabla) \mathbf{v}+\nabla \varphi=0
$$

In the Newtonian theory, the system is closed by the Poisson equation

$$
\nabla^{2} \varphi=\frac{3}{2} \mathcal{H}^{2} \Omega_{m} \delta_{N}
$$

Unlike the relativistic Poisson equation (35), which is only valid at first order, in the Newtonian case this equation is exact.

Combining the divergence of Equation (100) with the Poisson equation gives

$$
\frac{\partial(\nabla \cdot \mathbf{v})}{\partial \tau}+\mathcal{H} \nabla \cdot \mathbf{v}+\nabla \cdot(\mathbf{v} \cdot \nabla) \mathbf{v}+\frac{3}{2} \mathcal{H}^{2} \Omega_{m} \delta_{\mathrm{N}}=0 .
$$

One can define a Newtonian deformation tensor as $\vartheta_{\mathrm{N}}^{i j}=\partial^{i} v^{j}$, with trace $\vartheta_{\mathrm{N}}$. For an irrotational fluid, there is a velocity potential $\mathbf{v}=\partial^{i} v$, and we can expand

$$
\begin{aligned}
\nabla \cdot(\mathbf{v} \cdot \nabla) \mathbf{v} & =\partial_{i}\left(v^{j} \partial_{j}\right) v^{i}=\partial_{i} \partial^{j} v \partial_{j} \partial^{i} v+\partial^{j} v \partial_{j} \nabla^{2} v \\
& =\vartheta_{\mathrm{N} j}^{i} \vartheta_{\mathrm{N} i}^{j}+\partial^{j} v \partial_{j} \vartheta_{\mathrm{N}} .
\end{aligned}
$$

Introducing the Lagrangian time derivative, related to the Eulerian derivative through

$$
\frac{d}{d \tau}=\frac{\partial}{\partial \tau}+\mathbf{v} \cdot \nabla
$$

and using Equation (103), we can write Equations (99) and (100) as

$$
\begin{gathered}
\frac{d \delta_{\mathrm{N}}}{d \tau}+\left(1+\delta_{\mathrm{N}}\right) \vartheta_{\mathrm{N}}=0, \\
\frac{d \vartheta_{\mathrm{N}}}{d \tau}+\mathcal{H} \vartheta_{\mathrm{N}}+\vartheta_{\mathrm{N} j}^{i} \vartheta_{\mathrm{N} i}^{j}+\frac{3}{2} \mathcal{H}^{2} \Omega_{m} \delta_{\mathrm{N}}=0,
\end{gathered}
$$

i.e., the continuity equation and the Newtonian Raychaudhuri equation in Lagrangian form (Peebles 1980). The formal equivalence of these Newtonian evolution equations with their relativistic counterpart Equations (13) and (15) is evident, as long as one keeps in mind that a partial derivative with respect to the synchronous-comoving time $\tau$ in the relativistic case, corresponds to the convective Lagrangian derivative in the Newtonian case. The difference remains in the constraint equations (Matarrese et al. 1994), i.e., the energy constraint equation (16) and the momentum constraint equation (19) in the relativistic case, versus the Poisson equation (101). At first order, the equivalence of the dynamics is complete because the first-order Poisson equation, Equation (35), combines both relativistic constraint equations.

The equivalence of the equations allow us to establish a correspondence between Newtonian and relativistic variables, which we summarize as

$$
\begin{aligned}
\text { Newtonian Lagrangian } & \leftrightarrow \text { Relativistic comoving } \\
\frac{d}{d \tau} & \leftrightarrow \frac{\partial}{\partial \tau} \\
\partial^{i} \mathrm{v}_{j} & \leftrightarrow \vartheta_{j}^{i} \\
\delta_{\mathrm{N}} & \leftrightarrow \delta \\
\varphi_{\mathrm{IN}}^{(1)} & \leftrightarrow \frac{3}{5} \mathcal{R}_{\mathrm{c}} .
\end{aligned}
$$

Although the correspondence between deformation tensors and between density contrast is exact, that between metric potentials is only valid at first order in the matter-dominated era, where $\Phi_{\mathrm{IN}}=\varphi_{\mathrm{IN}}^{(1)}$. In addition, we note that the interpretation of $\vartheta_{j}^{i}$ is gauge-dependent; it is only in our synchronous-comoving gauge that it coincides with the extrinsic curvature, i.e., the deformation of the space slices. In general, in other gauges, $\vartheta_{i}^{j}$ will contain a Newtonian term $\partial_{i} v^{j}$ as well as post-Newtonian contributions from the metric (Bruni et al. 1992; I. Milillo et al. 2014, in preparation). 


\subsection{Initial Conditions for Numerical Simulations}

Our results suggest that numerical simulations of LSS formation based on Newtonian evolution equations can describe general relativistic evolution, even on super-horizon scales, in the case of irrotational flow in a $\Lambda \mathrm{CDM}$ cosmology, where the above dictionary should be used to interpret Newtonian variables in terms of their "true" relativistic meaning. In numerical codes, the first stage of the evolution of matter fluctuations is computed by solving the Newtonian equations of hydrodynamics at nonlinear orders in a perturbative expansion. However, we have seen that care needs to be taken when setting initial conditions such that they respect the nonlinear constraint equations of GR.

In Newtonian gravity, the Poisson equation is valid at all orders of perturbation. This is customarily used to account for primordial non-Gaussianity of local type (see, e.g., Dalal et al. 2008; Scoccimarro et al. 2012) through the expansion

$$
\delta_{\mathrm{IN}}=\frac{2}{3 \mathcal{H}_{\mathrm{IN}}^{2}} \nabla^{2} \varphi_{\mathrm{IN}}=\frac{2}{3 \mathcal{H}_{\mathrm{IN}}^{2}} \nabla^{2}\left[\Phi_{\mathrm{IN}}-f_{\mathrm{NL}} \Phi_{\mathrm{IN}}^{2}\right],
$$

where $\Phi_{\text {IN }}$ is a Gaussian first-order potential. ${ }^{5}$ The above constraint, or its Fourier counterpart, is imposed at some initial redshift, usually of the order $z\left(\tau_{\mathrm{IN}}\right) \sim 10^{2}$, in the matterdominated universe.

In the GR framework, the above equivalence is incomplete since the Poisson equation (35) is valid only for linear perturbations (Wands \& Slosar 2009). The above initial condition (107) disregards nonlinear GR contributions. The result in Equation (96) provides the correct constraint on large scales, consistent with GR. Therefore, the initial condition, at second order, in terms of the Gaussian potential $\Phi_{\mathrm{IN}}=(3 / 5) \mathcal{R}_{\mathrm{c}}$ yields the result

$$
\begin{aligned}
\frac{1}{2} \delta_{\mathrm{IN}}^{(2)}= & -\frac{4}{3 \mathcal{H}_{\mathrm{IN}}^{2}}\left[\left(f_{\mathrm{NL}}-\frac{5}{3}\right) \Phi_{\mathrm{IN}} \nabla^{2} \Phi_{\mathrm{IN}}\right. \\
& \left.+\left(f_{\mathrm{NL}}+\frac{5}{12}\right) \partial^{j} \Phi_{\mathrm{IN}} \partial_{j} \Phi_{\mathrm{IN}}\right] .
\end{aligned}
$$

Note that we have omitted here the particular part of the solution in Equation (96) since that is sub-dominant on largescales and at early times, and because it is generated by the subsequent Newtonian evolution. Initial conditions in the comoving-Poisson gauge have been presented as a solution for $\delta^{(2)}$ (Bartolo et al. 2010) or in terms of the constraint equations (Hidalgo et al. 2013).

In numerical simulations, the initial conditions are set by approximate solutions to the nonlinear equations. The codes fulfilling this task, dubbed initial condition generators, follow most commonly the Zel'dovich approximation (Zel'dovich 1970) or the more accurate second-order solution in Lagrangian coordinates (2LPT; e.g., Scoccimarro 1998). Our prescription for initial conditions sets a precedent to make the initial condition generators consistent with GR.

We show in the Appendix that the second-order density perturbation equation (108) includes a contribution in the squeezed limit equivalent to a primordial non-Gaussianity parameter $f_{\mathrm{NL}}=-5 / 3$. Equivalently, the final term in Equation (108) may be neglected when considering the statistics of virialized objects, corresponding to peaks of the matter density field. Around these

\footnotetext{
5 Often in the Newtonian context, a different sign convention is used (Wands 2010).
}

maxima, one could argue that $\nabla \Phi \approx 0$ (Dalal et al. 2008). Therefore, comparing with Equation (107), the relativistic corrections yield an effective contribution of magnitude $f_{\mathrm{NL}}=-5 / 3$.

\section{DISCUSSION}

Our starting point is the system of coupled nonlinear evolution equations for the inhomogeneous expansion, $\vartheta$, and density contrast, $\delta$, for an irrotational flow in GR in the synchronouscomoving gauge, adopting the spirit of the fluid-flow approach to cosmological perturbations. We have seen that these evolution equations correspond to the Newtonian evolution equations where we identify the comoving density with the Lagrangian density and the expansion with the divergence of the Eulerian velocity, $v$.

At first order in perturbative expansion, the correspondence $\vartheta \rightarrow \partial^{i} \partial_{i} v$ can be obtained by performing a gauge transformation from the synchronous-comoving gauge to the Poisson gauge. The equivalence at first order of the Newtonian theory with the relativistic equations for the matter density fluctuation in the comoving gauge and the velocity in the Poisson gauge has recently been discussed (Chisari \& Zaldarriaga 2011; Green \& Wald 2012) ${ }^{6}$ with particular emphasis in the study of the scaledependent bias of the LSS (Wands \& Slosar 2009; Baldauf et al. 2011; Bruni et al. 2012). In the present work, we have provided equations that extend the correspondence to nonlinear order and establish a framework in which the effects of primordial nonGaussianity in the LSS can be studied, keeping the consistency within GR.

We have presented solutions up to second order in a perturbative expansion about a background $\Lambda$ CDM cosmology. We believe that our derivation using the fluid-flow approach is more transparent than earlier derivations, revealing the essential role of the GR constraint equations, relating the initial density to the primordial curvature perturbation. We find what we believe to be a new, non-separable, second-order solution for the growing mode of the local density perturbation at a general point in the density field, with arbitrary shape coefficient, $\Sigma$ defined in Equation (67). This reduces to previously known separable solutions in the matter-dominated (Einstein-de Sitter) limit (Bartolo et al. 2005) or in the special case of planar symmetry $(\Sigma=1)$ (Bartolo et al. 2010). It would therefore be interesting to connect our results to alternative approaches to study nonlinear effects in the density field, in particular, attempts to consider the Zel'dovich approximation in GR (Russ et al. 1996; Matarrese \& Terranova 1996; Villa et al. 2011; Rampf \& Rigopoulos 2013) and 2LPT (Bernardeau et al. 2002). The essential difference between Newtonian theory and GR is action-at-a-distance versus causality, i.e., in the way non-locality comes in. The gradient of Equation (100) would lead to the evolution equation for $\vartheta_{\mathrm{Nj}}^{i}$, Equation (17), with the curvature terms replaced by the tidal field (Barrow \& Goetz 1989; Bruni et al. 2003). The Zel'dovich approximation results in truncating these equations, thereby reintroducing locality in the evolution system. It would be interesting to explore this method of solution of the nonlinear equations, which goes beyond our second-order perturbative expansion, in the GR context.

The general solution at second order includes the Newtonian nonlinear growing mode $D_{2+}\left(D_{2+} \propto D_{+}^{2}\right.$ in the matterdominated era), which is known to generate a non-zero galaxy

\footnotetext{
6 The relativistic effect of fluids with non-zero pressure has been explored by
} Christopherson et al. (2013). 
bispectrum (Jeong \& Komatsu 2009). The equivalence of the relativistic and Newtonian nonlinear evolution equations has been reported before (Matarrese \& Terranova 1996; Noh \& Hwang 2004), but there is also a second-order correction to the linearly growing mode, $\propto D_{+}$, due to the nonlinear constraint equations in GR (Bartolo et al. 2005; Bartolo et al. 2010). This needs to be included in the initial conditions used for N-body simulations which then use Newtonian equations of motion to follow the evolution of structure.

This intrinsic non-Gaussianity in GR leads to a galaxy bispectrum equivalent to a primordial non-Gaussianity parameter $f_{\mathrm{NL}}=-5 / 3$ in the squeezed limit (Verde \& Matarrese 2009). This result is obtained by using the primordial curvature perturbation, $\zeta$, to set initial conditions on scales larger than the horizon length at the start of the matter era. On smaller scales we would need to include the effect on the comoving curvature perturbation of evolution during the preceding radiation era. This is a more challenging calculation, especially if one considers the effect of photon pressure on the baryons before decoupling, almost certainly requiring a numerical calculation. Fortunately, numerical codes have recently been constructed to calculate the intrinsic non-Gaussianity in the CMB anisotropies from secondorder effects (Pitrou et al. 2010; Huang \& Vernizzi 2013; Su et al. 2012; Pettinari et al. 2013), and these codes should also be able to calculate the second-order density perturbation at the start of the matter era, and hence the expected galaxy bispectrum from second-order terms on intermediate scales.

To extend our GR calculations to higher order in a perturbative expansion would require us to include vorticity and gravitational waves. Although vector and tensor modes will inevitably be generated from first-order scalar perturbations (Matarrese et al. 1998; Ananda et al. 2007; Lu et al. 2008), they do not affect the (scalar) density perturbation at second order. Nonetheless, they do appear in the second-order metric and need to be included in a consistent, relativistic treatment of observable effects, such as frame-dragging (Bruni et al. 2014).

The authors are grateful to Rob Crittenden, Roy Maartens, Marc Manera, Sabino Matarrese, Francesco Pace and Cornelius Rampf for useful comments. J.C.H. is funded by CONACyT (program Estancias Posdoctorales y Sabáticas al Extranjero para la Consolidación de Grupos de Investigación). This work was supported by STFC grants ST/H002774/1 and ST/ $\mathrm{K} 00090 \mathrm{X} / 1$.

Note added in proof. We also thank Claes Uggla and John Wainwright, who contacted us after this paper was submitted, providing useful comments. Their paper (Uggla \& Wainwright 2013) presents an interesting alternative derivation of the second-order relativistic perturbation Equation (96) in a gaugeinvariant fashion.

\section{APPENDIX}

\section{SOLUTION IN EULERIAN COORDINATES}

In this appendix, we make explicit the correspondence between the second-order GR solutions Equation (96) and the well-known Newtonian version in Eulerian coordinates. In Section 4, we have shown how the equations relevant to our analysis match those of the Newtonian treatment when the following correspondences are assumed,

$$
\vartheta_{j}^{i} \rightarrow \partial^{i} \partial_{j} v \quad \text { and } \quad \frac{\partial}{\partial \tau} \rightarrow \frac{d}{d \tau}=\frac{\partial}{\partial \tau}+\partial^{i} v \partial_{i} .
$$

The time derivative transformation in the Newtonian limit from a Lagrangian or convective derivative, to the partial or Eulerian derivative, represents a change of the spatial coordinates. In the passive approach of cosmological perturbation theory (e.g., Malik \& Wands 2009), this is a change of spatial coordinate (or threading) for a fluid element

$$
\mathbf{x} \rightarrow \widetilde{\mathbf{x}}=\mathbf{x}-\xi
$$

leading to a first-order change in the three-velocity

$$
\mathbf{v}^{(1)} \rightarrow \widetilde{\mathbf{v}}^{(1)}=\mathbf{v}^{(1)}-\xi^{\prime}
$$

To transform from the synchronous-comoving gauge, where $\mathbf{v}^{(1)}=0$, to a new Eulerian gauge where $\widetilde{\mathbf{v}}^{(1)}=\nabla v_{E}$, we have

$$
\xi_{i}=-\int \partial_{i} v_{E} d \tau
$$

In particular, we choose $\xi$ such that $\nabla^{2} v_{E}=\vartheta$.

This gauge transformation does not affect the first-order density contrast, since scalar perturbations are invariant under first-order spatial gauge transformations: $\delta_{E}^{(1)}=\delta_{\text {sync }}^{(1)}$. However, at second-order, under a first-order spatial gauge transformation (Equation (A2)) we have (Matarrese et al. 1998; Malik \& Wands 2009)

$$
\delta^{(2)} \rightarrow \widetilde{\delta}^{(2)}=\delta^{(2)}+2 \xi^{i} \partial_{i} \delta^{(1)},
$$

hence the second-order density contrast in the Eulerian gauge is

$$
\delta_{E}^{(2)}=\delta^{(2)}-2 \partial_{i} \delta^{(1)} \int \partial^{i} v_{E} d \tau
$$

In terms of the first-order solutions Equations (56) and (57), which gives

$$
\int v_{E} d \tau=\int \nabla^{-2} \vartheta d \tau=-\left[f_{1}\left(\Omega_{m}\right)+\frac{3}{2} \Omega_{m}\right]^{-1} \frac{\mathcal{R}_{\mathrm{c}}}{\mathcal{H}^{2}},
$$

we find the Eulerian solution

$$
\delta_{E}^{(2)}=\delta^{(2)}+2\left[f_{1}\left(\Omega_{m}\right)+\frac{3}{2} \Omega_{m}\right]^{-2} \frac{\partial_{i} \mathcal{R}_{\mathrm{c}} \partial^{i} \nabla^{2} \mathcal{R}_{\mathrm{c}}}{\mathcal{H}^{4}} .
$$

It is clear that nonlinear terms resulting from the gauge transformation from Lagrangian to Eulerian density, will not modify the relativistic correction, proportional to $D_{+}$in Equation (95) at second order, but rather contributes an additional "Newtonian" term proportional to $D_{+}^{2}$.

We can compare this with the usual Newtonian solution in the matter-dominated limit $\left(\Omega_{m}=1\right)$ where the full Eulerian solution, Equations (96) with (A8), reduces to

$$
\begin{aligned}
& \delta_{E}^{(2)}(\mathbf{x}, \tau)=-\frac{24}{25 \mathcal{H}^{2}} \\
& \quad \times\left\{\left(f_{\mathrm{NL}}-\frac{5}{3}\right) \mathcal{R}_{\mathrm{c}} \nabla^{2} \mathcal{R}_{\mathrm{c}}+\left(f_{\mathrm{NL}}+\frac{5}{12}\right) \partial^{j} \mathcal{R}_{\mathrm{c}} \partial_{j} \mathcal{R}_{\mathrm{c}}\right\} \\
& \quad+\frac{8}{25 \mathcal{H}^{4}}\left\{\frac{5}{7}\left(\nabla^{2} \mathcal{R}_{\mathrm{c}}\right)^{2}+\frac{2}{7} \partial^{i} \partial_{j} \mathcal{R}_{\mathrm{c}} \partial_{i} \partial^{j} \mathcal{R}_{\mathrm{c}}+\partial_{i} \mathcal{R}_{\mathrm{c}} \partial^{i} \nabla^{2} \mathcal{R}_{\mathrm{c}}\right\} .
\end{aligned}
$$

Transforming to Fourier space, we obtain

$$
\delta_{E \mathbf{k}}^{(2)}=2 \int \frac{d^{3} \mathbf{k}_{1} d^{3} \mathbf{k}_{2}}{(2 \pi)^{3}} \delta_{D}\left(\mathbf{k}-\mathbf{k}_{1}-\mathbf{k}_{2}\right) F_{2}\left(\mathbf{k}_{1}, \mathbf{k}_{2}\right) \delta_{\mathbf{k}_{1}}^{(1)} \delta_{\mathbf{k}_{2}}^{(1)} .
$$


with the kernel

$$
\begin{aligned}
F_{2}\left(\mathbf{k}_{1}, \mathbf{k}_{2}\right)= & -\frac{3}{D_{+}} \\
& \times\left\{\left(f_{\mathrm{NL}}-\frac{5}{3}\right) \frac{k_{1}^{2}+k_{2}^{2}}{2 k_{1}^{2} k_{2}^{2}}+\left(f_{\mathrm{NL}}+\frac{5}{12}\right) \frac{\mathbf{k}_{1} \cdot \mathbf{k}_{2}}{k_{1}^{2} k_{2}^{2}}\right\} \\
& +\left\{\frac{5}{7}+\frac{2}{7} \frac{\left(\mathbf{k}_{1} \cdot \mathbf{k}_{2}\right)^{2}}{k_{1}^{2} k_{2}^{2}}+\frac{\mathbf{k}_{1} \cdot \mathbf{k}_{2}\left(k_{1}^{2}+k_{2}^{2}\right)}{2 k_{1}^{2} k_{2}^{2}}\right\} \quad(\mathrm{A} 11)
\end{aligned}
$$

The second line of this equation, which dominates at late times, reproduces exactly the second-order Newtonian solution in Eulerian coordinates, e.g., Equation (45) in Section 2.4 .2 of (Bernardeau et al. 2002).

The first line of this equation represents the nonlinear initial conditions in GR, including both primordial non-Gaussianity and intrinsic non-Gaussianity due to nonlinear constraints in GR. In the squeezed limit, $k_{1} \rightarrow 0$, the first term dominates, and we have

$$
F_{2} \rightarrow-\frac{3}{2 D_{+}}\left(f_{\mathrm{NL}}-\frac{5}{3}\right) \frac{1}{k_{1}^{2}},
$$

showing the effect of GR corrections as an effective shift in the value of $f_{\mathrm{NL}}, \Delta f_{\mathrm{NL}}=-5 / 3$.

\section{REFERENCES}

Ade, P., Aghanim, N., Armitage-Caplan, C., et al. 2013, arXiv:1303.5076V2 Ananda, K. N., Clarkson, C., \& Wands, D. 2007, PhRvD, 75, 123518 Baldauf, T., Seljak, U., Senatore, L., \& Zaldarriaga, M. 2011, JCAP, 10, 031 Bardeen, J. M. 1980, PhRvD, 22, 1882

Barrow, J. D., \& Goetz, G. 1989, COGra, 6, 1253

Bartolo, N., Komatsu, E., Matarrese, S., \& Riotto, A. 2004, PhR, 402, 103 Bartolo, N., Matarrese, S., Pantano, O., \& Riotto, A. 2010, CQGra, 27, 124009 Bartolo, N., Matarrese, S., \& Riotto, A. 2005, JCAP, 10, 010

Bernardeau, F., Colombi, S., Gaztanaga, E., \& Scoccimarro, R. 2002, PhR, 367,1

Bruni, M., Crittenden, R., Koyama, K., et al. 2012, PhRvD, 85, 041301

Bruni, M., Dunsby, P. K. S., \& Ellis, G. F. R. 1992, ApJ, 395, 34

Bruni, M., Maartens, R., \& Tsagas, C. G. 2003, MNRAS, 338, 785

Bruni, M., Matarrese, S., Mollerach, S., \& Sonego, S. 1997, CQGra, 14, 2585

Bruni, M., Thomas, D. B., \& Wands, D. 2014, PhRvD, 89, 044010

Buchert, T. 1992, MNRAS, 254, 729

Byrnes, C. T., \& Choi, K.-Y. 2010, AdAst, 2010, 724525

Carroll, S. M., Press, W. H., \& Turner, E. L. 1992, ARA\&A, 30, 499

Chisari, N. E., \& Zaldarriaga, M. 2011, PhRvD, 83, 123505

Christopherson, A. J., Hidalgo, J. C., \& Malik, K. A. 2013, JCAP, 01, 002

Dalal, N., Dore, O., Huterer, D., \& Shirokov, A. 2008, PhRvD, 77, 123514

Desjacques, V., \& Seljak, U. 2010, CQGra, 27, 124011

Ellis, G. F. R. 1971, in General Relativity and Cosmology, ed. R. K. Sachs, Proc. Int. School of Physics 'Enrico Fermi' (Varenna), Course XLVII (New York: Academic Press), 104
Ellis, G. F. R., \& Bruni, M. 1989, PhRvD, 40, 1804

Ellis, G. F. R., Maartens, R., \& H., M. M. A. 2012, Relativistic Cosmology (Cambridge: Cambridge Univ. Press)

Flender, S. F., \& Schwarz, D. J. 2012, PhRvD, 86, 063527

Giannantonio, T., Ross, A. J., Percival, W. J., et al. 2014, PhRvD, 89, 023511

Green, S. R., \& Wald, R. M. 2012, PhRvD, 85, 063512

Haugg, T., Hofmann, S., \& Kopp, M. 2012, arXiv:1211.0011

Hidalgo, J. C., Christopherson, A. J., \& Malik, K. A. 2013, JCAP, 08, 026

Huang, Z., \& Vernizzi, F. 2013, PhRvL, 110, 101303

Hwang, J.-c., Noh, H., \& Gong, J.-O. 2012, ApJ, 752, 50

Jeong, D., \& Komatsu, E. 2009, ApJ, 703, 1230

Kodama, H., \& Sasaki, M. 1984, PThPS, 78, 1

Koyama, K. 2010, CQGra, 27, 124001

Lahav, O., Lilje, P. B., Primack, J. R., \& Rees, M. J. 1991, MNRAS, 251,128

Landau, L. D., \& Lifshitz, E. M. 1975, The Classical Theory of Fields (Oxford: Pergamon)

Langlois, D., \& Vernizzi, F. 2005, PhRvL, 95, 091303

Linder, E. V., \& Cahn, R. N. 2007, APh, 28, 481

Lu, T. H.-C., Ananda, K., \& Clarkson, C. 2008, PhRvD, 77, 043523

Lyth, D. 1985, PhRvD, 31, 1792

Lyth, D. H., \& Liddle, A. R. 2009, The Primordial Density Perturbation (Cambridge: Cambridge Univ. Press)

Lyth, D. H., Malik, K. A., \& Sasaki, M. 2005, JCAP, 05, 004

Lyth, D. H., \& Rodríguez, Y. 2005, PhRvD, 71, 123508

Maldacena, J. M. 2003, JHEP, 05, 013

Malik, K. A., \& Wands, D. 2004, CQGra, 21, L65

Malik, K. A., \& Wands, D. 2009, PhR, 475, 1

Matarrese, S., Mollerach, S., \& Bruni, M. 1998, PhRvD, 58, 043504

Matarrese, S., Pantano, O., \& Saez, D. 1994, PhRvL, 72, 320

Matarrese, S., \& Terranova, D. 1996, MNRAS, 283, 400

Matarrese, S., \& Verde, L. 2008, ApJL, 677, L77

Meures, N. 2012, PhD thesis, Univ. Portsmouth

Misner, C. W., Thorne, K. S., \& Wheeler, J. A. 1973, Gravitation (San Francisco, CA: Freeman)

Noh, H., \& Hwang, J.-c. 2004, PhRvD, 69, 104011

Peebles, P. J. E. 1980, The Large-scale Structure of the Universe (Princeton, NJ: Princeton Univ. Press)

Pettinari, G. W., Fidler, C., Crittenden, R., Koyama, K., \& Wands, D. 2013, JCAP, 04, 003

Pitrou, C., Uzan, J.-P., \& Bernardeau, F. 2010, JCAP, 07, 003

Rampf, C., \& Rigopoulos, G. 2013, MNRAS, 430, L54

Russ, H., Morita, M., Kasai, M., \& Borner, G. 1996, PhRvD, 53, 6881

Scoccimarro, R. 1998, MNRAS, 299, 1097

Scoccimarro, R., Hui, L., Manera, M., \& Chan, K. C. 2012, PhRvD, 85, 083002

Su, S.-C., Lim, E. A., \& Shellard, E. 2012, arXiv:1212.6968

Tomita, K. 1967, PThPh, 37, 831

Tomita, K. 2005, PhRvD, 71, 083504

Uggla, C., \& Wainwright, J. 2013, arXiv:1312.1929

Verde, L., \& Matarrese, S. 2009, ApJL, 706, L91

Villa, E., Matarrese, S., \& Maino, D. 2011, JCAP, 08, 024

Wald, R. M. 1984, General Relativity (Chicago, IL: Univ. Chicago Press)

Wands, D. 2010, COGra, 27, 124002

Wands, D., Malik, K. A., Lyth, D. H., \& Liddle, A. R. 2000, PhRvD, 62, 043527

Wands, D., \& Slosar, A. 2009, PhRvD, 79, 123507

Wang, L.-M., \& Steinhardt, P. J. 1998, ApJ, 508, 483

Zel'dovich, Y. 1970, A\&A, 5, 84
7 Note that in Bernardeau et al. (2002) the perturbative expansion of nonlinear variables does not carry the usual Taylor-expansion numerical factors. Hence the factor of two included in our definition of $F_{2}$ in Equation (A11). 